\section{EF-07 CURLI AMYLOIDS/DNA COMPLEXES FROM BACTERIAL BIOFILMS BREAK TOLERANCE IN MURINE LUPUS BY TRIGGERING BCR/TLR SIGNALING IN B CELLS}

\footnotetext{
${ }^{1,2}$ Michael H Lee, ${ }^{2}$ Sarah A Tursi, ${ }^{2}$ Lauren K Nicastro, ${ }^{3}$ Roberto Caricchio, ${ }^{2}$ Çagla Tükel,

1,2 Stefania Gallucci*. ${ }^{1}$ Laboratory of Dendritic Cell Biology; ${ }^{2}$ Department of Microbiology and Immunology, Lewis Katz School of Medicine, Temple University; ${ }^{3}$ Division of Rheumatology, Department of Medicine, Lewis Katz School of Medicine, Temple University, Philadelphia, Pennsylvania 19140, USA
}

\subsection{6/lupus-2018-Ism.87}

Background The pathogenesis of Systemic Lupus Erythematosus (SLE) is multifactorial with genetic make-up and environmental triggers considered major players. Among the environmental triggers, infections are a major cause of morbidity and mortality in SLE patients. Bacteremia is often overlooked in SLE patients and soft tissue abscesses, bloodstream infections, and sepsis are more common in SLE patients, suggesting that frequent exposures to microbial products may trigger flares in lupus. We have recently shown that a bacterial amyloid termed curli, expressed in the multicellular communities (biofilms) by many bacteria including E. coli, plays a major role in triggering lupus autoimmunity during infection. Curli bind bacterial or eukaryotic DNA and form curli/DNA complexes that strongly activate innate immunity. When given systemically, curli/DNA complexes and infections with curli-expressing $E$. coli trigger production of anti-dsDNA and anti-chromatin autoAbs in lupus prone mice and in wild type mice. This affect was diminished in TLR2 or TLR9 deficient mice. Curli/DNA complexes activate dendritic cells and macrophages. We have now focused on the effects of curli/DNA complexes on $B$ cells.

Methods Young wild type C57BL/6 mice, lupus prone Sle1,2,3 mice and $3 \mathrm{H} 9$ mice were used. Splenic B cells were stained by flow cytometry for in vivo experiments. For in vitro experiments, B cells were sorted by positive selection with CD45R (B220) Miltenyi Biotec micro bead or Easysep B cell isolation kit (Stem cell) supplemented with anti-CD43-Biotin. B cell purity (>98\%), proliferation, viability, activation markers, surface antibodies and signaling were measured by CFSE dilution (Promokine), 7AAD and antibodies by Flow cytometry, Western Blot analysis and qRT-PCR.

Results We found that curli/DNA complexes polyclonally activate $\mathrm{B}$ cells in vivo in wildtype mice, lupus-prone mice and $3 \mathrm{H} 9$ mice, the latter expressing an anti-DNA Ig heavy chain and whose $\mathrm{B}$ cells are normally tolerized. Curli/DNA complexes can also activate $\mathrm{B}$ cells in vitro in the absence of $\mathrm{T}$ cell help. The induction of non-canonical $N F \kappa B$ in the absence of $\mathrm{T}$ cell help suggests that the fibrillar structure of curli/DNA complexes can cross-link BCRs, some recognizing DNA. Interestingly, curli/DNA complexes also induce isotype switching and aicda, the master regulator of class switch recombination, in the absence of $\mathrm{T}$ cells help in vitro.

Conclusions Our results suggest that curli/DNA complexes may induce anti-DNA antibody production by simultaneous BCR/ TLR signaling, which leads to B cell antibody production in the absence of $\mathrm{T}$ cell help.

Acknowledgements We would like to thank the Lupus Research Alliance and the NIH NIAID (R21AI119947) for supporting our work.

\section{Genetics, Genomics and Epigenetics}

\section{GG-01 HYPOMETHYLATION OF STAT1 AND HLA-DRB1 IN CD8+ T CELLS IS ASSOCIATED WITH TYPE-I INTERFERON- DEPENDENT HLA-DRB1 OVEREXPRESSION AND ACTIVATION OF AUTOLOGOUS CD4+ T CELLS IN SYSTEMIC LUPUS ERYTHEMATOSUS}

Shaylynn Miller, Patrick Coit, Elizabeth Gensterblum-Miller, Paul Renauer, Nathan C Kilian, Mark Schonfeld, Pei-Suen Tsou, Amr H Sawalha*. Division of Rheumatology, Department of Internal Medicine, University of Michigan, Ann Arbor, Michigan, USA

\subsection{6/lupus-2018-Ism.88}

Background Systemic lupus erythematosus is a chronic autoimmune disease characterized by epigenetic dysregulation, and increased autoantibody and type-I interferon (IFN) production. The goal of this study was to explore possible pathogenic roles of CD8 $+\mathrm{T}$ cells in lupus through characterizing DNA methylation changes.

Methods Genome-wide DNA methylation of lupus and age, sex and ethnicity-matched control CD8 + T cells was measured using the Infinium MethylationEPIC arrays. Data preprocessing and statistical analysis of differentially methylated $\mathrm{CpG}$ sites was performed using GenomeStudio (Illumina). Gene ontology analysis of differentially methylated sites was performed with DAVID. Measurement of HLA-DRB1 expression on the surface of lupus and control CD8 $+\mathrm{T}$ cells with and without interferon alpha (IFN $\alpha$ ) was performed by flow cytometry. Co-incubation of IFN $\alpha$-treated CD8 $+\mathrm{T}$ cells from lupus patients and controls with autologous naïve $\mathrm{CD} 4+\mathrm{T}$ cells to assess effects on CD4+ T cell stimulation were performed. CD8 + T cell mRNA levels normalized to $\beta$-actin were quantified by qPCR.

Results Lupus CD8 + T cells had 188 hypomethylated CpG sites compared to healthy matched controls. Among the most demethylated were sites associated with HLA-DRB1 $(\Delta \beta=-0.33)$ and STAT1 $(\Delta \beta=-0.15)$. The proportion of $\mathrm{CD} 8+\mathrm{T}$ cells expressing HLA-DRB1 was significantly higher in lupus compared to controls. IFN $\alpha$ treatment upregulated cell surface expression of HLA-DRB1 on CD8 $+\mathrm{T}$ cells of lupus patients but not healthy controls. Co-incubation of naïve CD4 $+\mathrm{T}$ cells with IFN $\alpha$-treated autologous CD8 $+\mathrm{T}$ cells led to increased expression of the stimulation marker CD69 on CD4+ T cells in lupus patients, but not in healthy controls. This effect can be abrogated using HLA-DR blocking antibodies. Lupus and control CD8 $+\mathrm{T}$ cells significantly increased STAT1 mRNA levels after treatment with IFN $\alpha$. The expression of CIITA, a key interferon/STAT1 dependent MHC-class II regulator, is only induced by IFN $\alpha$ in lupus CD8 + T cells, but not healthy controls.

Conclusions HLA-DRB1 and STAT1 loci are hypomethylated and epigenetically poised for overexpression in lupus CD8 $+\mathrm{T}$ cells in the presence of type-I interferon. IFN $\alpha$-treated lupus $\mathrm{CD} 8+\mathrm{T}$ cells stimulate autologous $\mathrm{CD} 4+\mathrm{T}$ cells in vitro and blocking of HLA-DR on CD8 $+\mathrm{T}$ cells can reduce this effect. These data suggest a possible pathogenic role for $\mathrm{CD} 8+\mathrm{T}$ cells in lupus that is dependent upon a high type-I interferon environment and epigenetic priming.

Acknowledgements This work was supported by the National Institute of Allergy and Infectious Diseases of the National Institutes of Health grant number R01AI097134. 